\title{
Shock wave elimination/reduction by optimal coordination of variable speed limits*
}

\author{
P. Breton, A. Hegyi, B. De Schutter, and H. Hellendoorn
}

If you want to cite this report, please use the following reference instead:

P. Breton, A. Hegyi, B. De Schutter, and H. Hellendoorn, "Shock wave elimination/reduction by optimal coordination of variable speed limits," Proceedings of the IEEE 5th International Conference on Intelligent Transportation Systems (ITSC'02), Singapore, pp. 225-230, Sept. 2002. 


\title{
Shock Wave Elimination/Reduction by Optimal Coordination of Variable Speed Limits
}

\author{
P. Breton, A. Hegyi, B. De Schutter, H. Hellendoorn
}

\begin{abstract}
We present a model predictive control (MPC) approach to optimally coordinate variable speed limits for highway traffic. The basic idea is that dynamic speed limits can create traffic conditions where shock waves can damp out faster. The control objective is to minimize the total time that vehicles spend in the network. For the prediction of the evolution of the traffic flows in the network we use an adapted version of the METANET model that takes the variable speed limits into account. The coordinated control results in a network with less congestion, a higher outflow, and a lower total time spent. In addition, the receding horizon approach of MPC results in an adaptive, on-line control strategy that automatically takes changes in the system parameters into account.
\end{abstract}

Index Terms-traffic control, coordinated control, variable speed limits, model predictive control

\section{INTRODUCTION}

A $S$ the number of vehicles and the need for transportation grow, cities around the world face considerable traffic congestion problems: almost every weekday morning and evening during rush hours the saturation point of the highways and the main roads in and around the city is attained. Traffic jams do not only cause considerable costs due to unproductive time losses, but they also augment the possibility of accidents, and they have a negative impact on the environment and on the quality of life. On the short term the most effective measures in the battle against traffic congestion seem to be a selective construction of new roads and a better control of traffic by dynamic traffic management measures. We will concentrate on the latter option.

In practice, dynamic traffic management usually operates based on local data only. However, considering the effect of the measures on the network level has many advantages compared to local control. E.g., solving a local congestion may have as a consequence that the vehicles run faster into another downstream congestion because of the improved flow, whereas still the same number of vehicles have to pass the bottleneck (which has a fixed capacity). So, the average travel time in the network will still be the same. Another reason is that in a dense network the effect of a local control measure could also influence the traffic flows in more distant parts of the network: an improved (or delayed) flow could cause (or

P. Breton, A. Hegyi, B. De Schutter, H. Hellendoorn are with the Control Systems Engineering group, Faculty of Information Technology and Systems, Delft University of Technology, P.O. Box 5031, 2600 GA Delft, The Netherlands. E-mail: \{a.hegyi,b.deschutter,j.hellendoorn\} dits.tudelft.nl prevent) congestion somewhere else in the network. Furthermore, if dynamic origin-destination (OD) data is available, control on the network level can take the predicted flows in the network into account. Local controllers are not able to use OD information because the traffic flow arriving at the local controller depends on the actions of other controllers in the network, which are unknown. Another source of degradation of network performance is that congestion might block traffic routes that do not go over the cause of congestion (e.g., a motorway mainstream blocked by congestion spilling back from an off-ramp).

To address the problems sketched above a control strategy that operates on the network level is needed, i.e., there should be a network-wide coordination of control measures, based on global data. Since the effect of a control measure on more distant locations might only be visible after some time, a prediction of the network evolution is also needed to achieve optimal network control. To predict the effects of a control measure several techniques can be used, such as case-based reasoning, rule-based systems, or model-based prediction. In this paper we opt for the latter approach. More specifically, we use the METANET traffic simulation model [7], [13] for the predictions, and we apply a model predictive control framework (MPC) [3], [11] to find the optimal control inputs. In practice, the prediction model used by the controller is always different from the real system, and the disturbances are only partially known. Model predictive control is known to perform well when this occurs.

The main control objective is to minimize the total time spent (TTS) by the vehicles in the network, but we will add an extra term to the objective function to penalize abrupt changes in the control signal. Papageorgiou [12] showed that, under the condition that the network inflow is known or can be predicted accurately, minimizing TTS is equivalent to maximizing the time-weighted outflow of the network. That means that a controller that minimizes TTS will tend to maximize the outflow as soon as possible.

In literature basically two views on the use of speed limits can be found. The first emphasizes the homogenization effect [1], [2], [5], [16], [15], whereas the second is more focused on the prevention of traffic breakdown [9], [10], [4]. The idea of homogenization is that speed limits reduce the speed differences between vehicles by which a higher (and safer) flow can be achieved. The homogenization approach typically uses speed limits that are close to the critical speed (the speed that corresponds to the maximal flow. See Figure 1). 


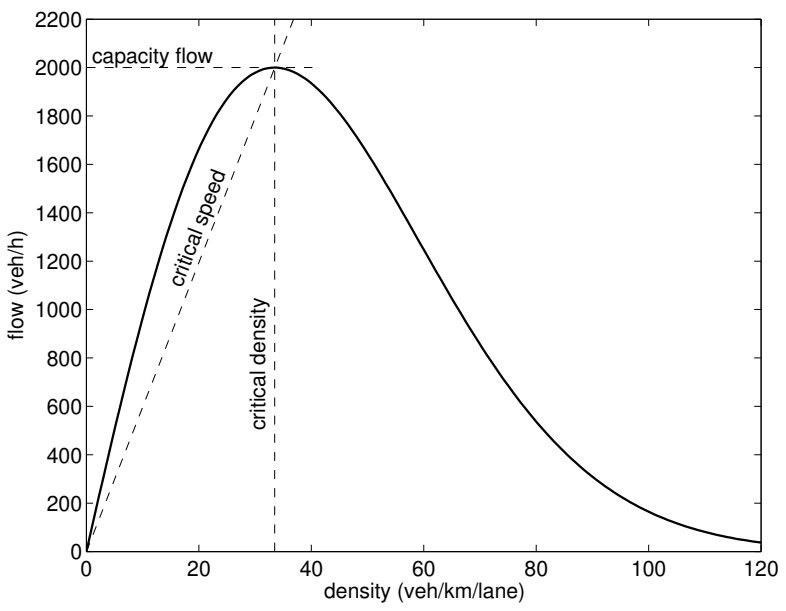

Figure 1. A typical example of the fundamental diagram. The critical speed is the speed that corresponds to maximum flow.

The traffic breakdown prevention approach focuses more on preventing too high densities, and it also allows lower than critical speed limits. The results in [16] indicate that the effect of homogenization on motorway performance is negligible; however, a positive safety effect can be expected. To the authors' best knowledge there are currently no published results available of experiments in connection with using speed limits to prevent traffic breakdown. Currently, the Dutch Ministry of Transport, Public Works and Water Management is preparing an experiment in the DYVERS project where the reduction of congestion by dynamic speed limits is studied (in this experiment the speed limits will be enforced).

Several control methodologies are used in literature to find a control law for speed control, such as multi-layer control [12], sliding-mode control [9], [10], or optimal control [1], [2]. In [5] the optimal control is approximated by a neural network (NN) in a rolling horizon framework. The authors mention online optimization (instead of a static control law represented by the $\mathrm{NN}$ ) as an option, but they prefer the NN approach because of its speed. In this paper we demonstrate the feasibility of online optimization. The advantage of this approach is that it is able to adapt to changing traffic conditions.

Most of the models used in literature represent the speed limits by a factor that downscales the fundamental diagram. This can give too optimistic results (see Section III-B2), and therefore we introduce another equation. We also introduce an equation to express the difference in the drivers anticipation to increasing or decreasing downstream densities.

The organization of the rest of the paper is as follows. In Section II the problem and the basic idea of the solution of moving jams is described. In Section III the basic ingredients of model predictive control are introduced, and the prediction model including the extensions is presented. The proposed control method is applied to a benchmark problem in Section IV. Finally the conclusions and topics for future research are stated in Section V.

\section{PROBLEM DESCRIPTION}

It is well known (see, e.g., [6]) that some type of traffic jams move upstream with approximately $15 \mathrm{~km} / \mathrm{h}$. These jams can remain stationary for a long time, so every vehicle that enters the motorway upstream of the jammed area will have to pass through the jammed area, which increases the travel time.

Lighthill and Whitham introduced the term shock wave for waves that are formed by several waves running together. At the shock wave fairly large reductions in velocity occur very quickly. In this paper we will use the term "shock wave" for any wave (the moving jammed areas) and not distinguish between waves and shock waves, because in practice any wave is undesired. Besides the increased travel time another disadvantage of these shock waves is that they are potentially unsafe.

To eliminate the shock wave speed limits can be used in the following way. On some sections upstream of the shockwave the speed limits are reduced and consequently the inflow of the jammed area is reduced. Another view on what happens is the following. The decreased speed limits create a low density wave (with a density lower than it would be in the uncontrolled situation) that propagates downstream. This low density wave meets the shockwave and compensates its high density, which reduces or eliminates the shock wave. A necessary condition is that the steady state flow (without shock wave) is below capacity, so that the speed limits do not create a traffic breakdown. We will see that this rest capacity does not have to be large.

A point of criticism could be that the approach reduces the shock wave, but at the cost of creating new shock waves upstream of the sections controlled by speed limits. However, if the speed limits are optimized properly, they will never create shock waves that give rise to higher delays than in the uncontrolled case. The speed limits will delay the traffic, but only temporarily and on the average the flow will be higher than if shock wave would have passed along the whole motorway stretch. So the inflow of the network will be decreased by the speed limits only for a short period of time, and TTS in the network will be lower. A necessary condition is that the speed limits are optimized properly. In the following sections we will demonstrate how this optimization can be achieved.

\section{APPROACH}

\section{A. Model Predictive Control}

We use a model predictive control (MPC) scheme to solve the problem of optimal coordination of speed limits. At each time step $k$ the optimal control signal is computed (by numerical optimization) over a prediction horizon $N_{\mathrm{p}}$. A control horizon $N_{\mathrm{c}}\left(<N_{\mathrm{p}}\right)$ is selected to reduce the number of variables and to improve the stability of the system. After the control horizon has been passed the control signal is usually taken to be constant. A rolling horizon strategy is used, which means that at each time step only the first sample of the 


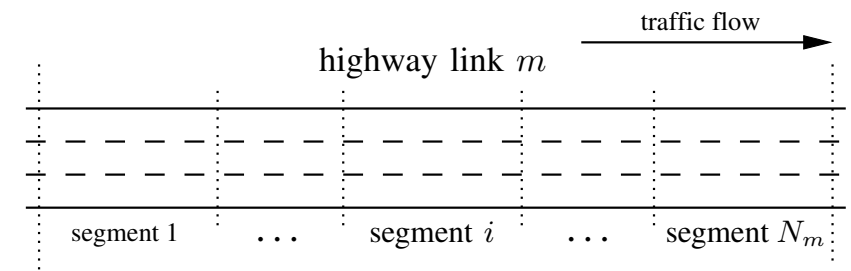

Figure 2. In the METANET model a motorway link is divided into segments.

optimal control signal is applied to the system; afterward the time axis is shifted one sample step, the model is updated, and the procedure is restarted.

This rolling horizon approach results in an on-line adaptive control scheme that allows us to take changes in the system or in the system parameters into account by regularly updating the model of the system or the predicted demands as new measurements from the traffic sensors become available. For more information see [3], [11] and the references therein.

\section{B. Prediction model}

The MPC procedure includes a prediction of the network evolution as a function of the current state and a given control input. For this prediction we use a slightly modified version of the METANET model [7], [13]. For the sake of brevity, we describe only those parts of the model that are relevant for interpreting and understanding the simulation results of our benchmark network (see Section IV).

1) Original METANET model: The METANET model represents a network as a directed graph with the links corresponding to highway stretches. Each motorway link has uniform characteristics, i.e., no on-ramps or off-ramps and no major changes in geometry. Each link $m$ is divided into $N_{m}$ segments of length $L_{m}$ (see Figure 2). Each segment $i$ of link $m$ is characterized by the traffic density $\rho_{m, i}(k)$ (veh/lane $\left./ \mathrm{km}\right)$, the mean speed $v_{m, i}(k)(\mathrm{km} / \mathrm{h})$, and the traffic volume or flow $q_{m, i}(k)(\mathrm{veh} / \mathrm{h})$, where $k$ indicates the time instant $t=k T$, and $T$ is the time step used for the simulation of the traffic flow (typically $T=10 \mathrm{~s}$ ).

The following equations describe the evolution of the network over time. The outflow of each segment is equal to the density multiplied by the mean speed and the number of lanes on that segment (denoted by $\lambda_{m}$ ):

$$
q_{m, i}(k)=\rho_{m, i}(k) v_{m, i}(k) \lambda_{m} .
$$

The density of a segment equals the previous density plus the inflow from the upstream segment, minus the outflow of the segment itself (conservation of vehicles):

$$
\rho_{m, i}(k+1)=\rho_{m, i}(k)+\frac{T}{L_{m} \lambda_{m}}\left(q_{m, i-1}(k)-q_{m, i}(k)\right) .
$$

The mean speed equals the previous mean speed plus a relaxation term that expresses that the drivers try to achieve a desired speed $V(\rho)$, a convection term that expresses the speed increase (or decrease) caused by the inflow of vehicles, and an anticipation term that expresses the speed decrease (increase) as drivers experience a density increase (decrease) downstream:

$$
\begin{gathered}
v_{m, i}(k+1)=v_{m, i}(k)+\frac{T}{\tau}\left(V\left(\rho_{m, i}(k)\right)-v_{m, i}(k)\right)+ \\
\frac{T}{L_{m}} v_{m, i}(k)\left(v_{m, i-1}(k)-v_{m, i}(k)\right)- \\
\frac{\nu T}{\tau L_{m}} \frac{\rho_{m, i+1}(k)-\rho_{m, i}(k)}{\rho_{m, i}(k)+\kappa},
\end{gathered}
$$

where $\tau, \nu$ and $\kappa$ are model parameters, and with

$$
V\left(\rho_{m, i}(k)\right)=v_{\text {free }, m} \exp \left[-\frac{1}{a_{m}}\left(\frac{\rho_{m, i}(k)}{\rho_{\text {crit }, m}}\right)^{a_{m}}\right],
$$

with $a_{m}$ a model parameter, and where the free-flow speed $v_{\text {free, } m}$ is the average speed that drivers assume if traffic is flowing freely, and the critical density $\rho_{\text {crit }, m}$ is the density at which the traffic flow becomes unstable.

Origins are modeled with a simple queue model. The length of the queue $w_{o}(k)$ equals the previous queue length plus the demand $^{1} d_{o}(k)$, minus the outflow $q_{o}(k)$ :

$$
w_{o}(k+1)=w_{o}(k)+T\left(d_{o}(k)-q_{o}(k)\right) .
$$

The outflow depends on the traffic conditions on the motorway and the capacity of the origin. The flow $q_{o}(k)$ is the minimum of the demand and the maximal flow that can enter the motorway given the mainstream conditions:

$$
q_{o}(k)=\min \left[d_{o}(k)+\frac{w_{o}(k)}{T}, Q_{o} \frac{\rho_{\max }-\rho_{\mu, 1}(k)}{\rho_{\max }-\rho_{\text {crit }, \mu}}\right],
$$

where $Q_{o}$ is the on-ramp capacity (veh/h) under free-flow conditions, $\rho_{\max }$ is the maximum density, and $\mu$ the index of the link to which the on-ramp is connected.

The coupling equations to connect links are as follows. Every time there is a major change in the link parameters or there is a junction or a bifurcation, a node is placed between the links. This node provides the incoming links with a downstream density, and the leaving links with an inflow and a upstream speed. The flow that enters node $n$ is distributed among the leaving links according to

$$
\begin{aligned}
Q_{n}(k) & =\sum_{\mu \in I_{n}} q_{\mu, N_{\mu}}(k) \\
q_{m, 0}(k) & =\beta_{n}^{m}(k) \cdot Q_{n}(k) \quad \forall m \in O_{n},
\end{aligned}
$$

where $Q_{n}(k)$ is the total flow that enters the link at time $k$, $I_{n}$ is the set of links that enter node $n, \beta_{n}^{m}(k)$ are the turning rates (the fraction of the total flow through node $n$ that leaves via link $m$ ), and $q_{m, 0}(k)$ is the flow that leaves node $n$ via link $m$.

When node $n$ has more than one leaving link, the virtual downstream density $\rho_{m, N_{m}+1}(k)$ of entering link $m$ is given by

$$
\rho_{m, N_{m}+1}(k)=\frac{\sum_{\mu \in O_{n}} \rho_{\mu, 1}^{2}(k)}{\sum_{\mu \in O_{n}} \rho_{\mu, 1}(k)},
$$

\footnotetext{
${ }^{1}$ Just as in [7], [8], [14] we assume that the demand is independent of any control actions taken in the network. Otherwise, a larger network should be considered.
} 
where $O_{n}$ is the set of links leaving node $n$.

When node $n$ has more than one entering link, the virtual upstream speed $v_{m, 0}(k)$ of leaving link $m$ is given by

$$
v_{m, 0}(k)=\frac{\sum_{\mu \in I_{n}} v_{\mu, N_{\mu}}(k) \cdot q_{\mu, N_{\mu}}(k)}{\sum_{\mu \in I_{n}} q_{\mu, N_{\mu}}(k)} .
$$

In addition, when there is no entering link (but a mainstream origin) we assume - as a boundary condition - that the speed of the (virtual) entering link equals the speed of the first segment

$$
v_{m, 0}(k)=v_{m, 1}(k) .
$$

This is a good approximation of the speed behavior when there are enough $(\geq 3)$ uncontrolled upstream segments.

2) Extensions: Since the original METANET model does not describe the effect of speed limits, we have slightly modified the equation for the desired speed (3) to incorporate speed limits. The second extension is regarding the modeling of the different nature of a mainstream origin opposed to an on-ramp origin. The third extension considers the different effect of the downstream density gradient on the speed (anticipation term in (2)) when it is positive or negative.

Note that the MPC approach is generic and will find the optimal speed limits independent from the model that is used (e.g. way that speed limits enter the model).

In some publications the effect of the speed limit is expressed by scaling down the desired speed (a function of density) by $v_{\text {ctrl }} / v_{\text {free, } m}$. This changes the whole speeddensity diagram, also for the states where the speed would otherwise be lower than the value of the speed limit. This means, for example, that if the free flow speed is $120 \mathrm{~km} / \mathrm{h}$ and the displayed speed limit is $100 \mathrm{~km} / \mathrm{h}$ then it is assumed that the speed and flow of the traffic are reduced even when the vehicles are traveling at $80 \mathrm{~km} / \mathrm{h}$. Furthermore, scaling down the desired speed also reduces the capacity, while there is no reason to assume that a speed limit above the critical speed (speeds where the flow has not reached capacity yet) would reduce the capacity of the road (see Figure 1). These assumptions are rather unrealistic, and they exaggerate the effect of speed limits. However, to get a more realistic model for the effects of the speed limits, we assume that the desired speed is the minimum of the following two quantities: the desired speed based on the experienced density, and the desired speed caused by the speed limit displayed on the variable message sign (VMS):

$$
\begin{aligned}
& V\left(\rho_{m, i}(k)\right)= \\
& \quad \min \left(v_{\mathrm{ctrl}, m, i}(k), v_{\text {free }, m} \exp \left[-\frac{1}{a_{m}}\left(\frac{\rho_{m, i}(k)}{\rho_{\text {crit }, m}}\right)^{a_{m}}\right]\right),
\end{aligned}
$$

where $v_{\mathrm{ctrl}, m, i}(k)$ is the speed limit imposed on segment $i$, link $m$, at time $k$.

To express the different nature of a mainstream origin link $o$ compared to a regular on-ramp (the queue at a mainstream origin is in fact an abstraction of the sections upstream of the origin of part of motorway network that we are modeling), we use a modified version of (4) with another flow constraint, because the inflow of a segment (and thus the outflow of the mainstream origin) can be limited by an active speed limit or by the actual speed on the first segment (when either of them is lower than the speed at critical density). We assume that the maximal flow equals the flow that follows from the speedflow relationship from (1) and (3) with the speed equal to the speed limit or the actual speed on the first segment whichever is smaller. So if $o$ is the origin of link $\mu$, then we have

$$
q_{o}(k)=\min \left[d_{o}(k)+\frac{w_{o}(k)}{T}, q_{\lim , \mu, 1}(k)\right] \text {, }
$$

where $q_{\lim , \mu, 1}(k)$ is the maximal inflow determined by the limiting speed in the first segment of link $\mu$ :

$$
\begin{aligned}
& q_{\lim , \mu, 1}(k)= \\
& \left\{\begin{aligned}
\lambda_{\mu} v_{\mathrm{ctrl}, \mu, 1}(k) \rho_{\mathrm{crit}, \mu}[- & \left.a_{\mu} \ln \left(\frac{v_{\lim , \mu, 1}(k)}{v_{\mathrm{free}, m}}\right)\right]^{\frac{1}{a_{\mu}}} \\
& \text { if } v_{\mathrm{ctrl}, \mu, 1}(k)<V\left(\rho_{\mathrm{crit}, \mu}\right) \\
q_{\mathrm{cap}, \mu} & \text { if } v_{\mathrm{ctrl}, \mu, 1}(k) \geq V\left(\rho_{\mathrm{crit}, \mu}\right),
\end{aligned}\right.
\end{aligned}
$$

where $v_{\lim , \mu, 1}(k)=\min \left(v_{\operatorname{ctrl}, \mu, 1}(k), v_{\mu, 1}(k)\right)$ is the speed that limits the flow, and $q_{\mathrm{cap}, \mu}=\lambda_{\mu} V\left(\rho_{\text {crit }, \mu}\right) \rho_{\mathrm{crit}, \mu}$ is the capacity flow.

Since the effect of a higher downstream density is usually stronger than the effect of a lower downstream density, we distinguish between these two cases. The sensitivity of the speed to the downstream density is expressed by parameter $\nu$. In (2) $\nu$ is a global parameter and has the same value for all segments, however, here we and take different values for $\nu_{m, i}(k)$ for when the downstream density is higher or lower than the density in the actual segment.

$$
\nu_{m, i}(k)= \begin{cases}\nu_{\text {high }} & \text { if } \rho_{m, i+1}(k) \geq \rho_{m, i}(k) \\ \nu_{\text {low }} & \text { if } \rho_{m, i+1}(k)<\rho_{m, i}(k) .\end{cases}
$$

\section{Objective function}

We consider the following objective function:

$$
\begin{gathered}
J(k)=T \sum_{l=k}^{k+N_{\mathrm{p}}-1}\left\{\sum_{m, i} \rho_{m, i}(l) L_{m} \lambda_{m}+\sum_{o} w_{o}(l)\right\}+ \\
a_{\text {speed }} \sum_{l=k}^{k+N_{\mathrm{c}}-1} \sum_{(m, i) \in I_{\text {speed }}}\left(\frac{v_{\mathrm{ctrl}, m, i}(l)-v_{\mathrm{ctrl}, m, i}(l-1)}{v_{\text {free }, m}}\right)^{2},
\end{gathered}
$$

where $I_{\text {speed }}$ is the set of pairs of indices $(m, i)$ of the links and segments where speed control is applied. This objective function contains a term for the TTS, and a term that penalizes abrupt variations in the speed limit control signal. The variation term is weighted by the nonnegative weight parameter $a_{\text {speed }}$. 


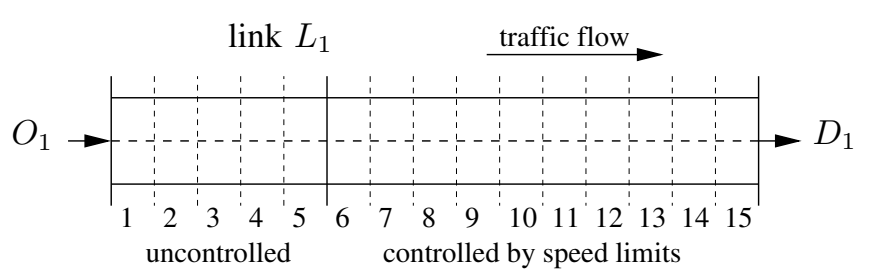

Figure 3. The benchmark network includes five uncontrolled and ten controlled sections with speed limits. Each segment has a length of $1 \mathrm{~km}$.

\section{Tuning of $N_{\mathrm{p}}$ and $N_{\mathrm{c}}$}

In conventional MPC heuristic tuning rules have been developed to select appropriate values for $N_{\mathrm{p}}$ and $N_{\mathrm{c}}$ (see [11]). However, these rules cannot be straightforwardly applied the traffic flow control framework presented above.

If we take the prediction horizon $N_{\mathrm{p}}$ shorter than the typical travel time in the network (under presence of a shock wave), then the effect of the vehicles that are influenced by the current control measure and - as a consequence - have an effect on the network performance before they exit the network, will not be taken into account. Furthermore, a control action may affect the network state (by improved flows, etc.) even when the actually affected vehicles have already exited the network. On the other hand, $N_{\mathrm{p}}$ should not be too large because of the computational complexity of the MPC optimization problem. So based on this heuristic reasoning we select $N_{\mathrm{p}}$ to be about the typical travel time in the network when a shock wave is present. For the control horizon $N_{\mathrm{c}}$ we will select a value that represents a trade-off between the computational effort and the performance.

\section{A BENCHMARK PROBLEM}

In order to illustrate the control framework presented above we will now apply it to a motorway link equipped with variable speed signs.

\section{A. Set-up}

The benchmark network consists of one origin, one freeway link, and one destination (Figure 3). $O_{1}$ is the mainstream origin and has two lanes with a capacity of $2000 \mathrm{veh} / \mathrm{h}$ each. The motorway link $L_{1}$ follows with two lanes, and is $15 \mathrm{~km}$ long consisting of fifteen segments of $1 \mathrm{~km}$ each. Segments 1-5 are uncontrolled, segments 6-15 are equipped with a VMS where speed limits can be set. The choice for the five uncontrolled segments was made to be sure that boundary condition of equation (7) does not play a dominant role. Link $L_{1}$ ends in destination $D_{1}$. We use the same network parameters as in [7]: $T=10 \mathrm{~s}, \tau=18 \mathrm{~s}, \kappa=40 \mathrm{veh} / \mathrm{lane} / \mathrm{km}$, $\rho_{\max }=180 \mathrm{veh} / \mathrm{lane} / \mathrm{km}, \rho_{\text {crit }}=33.5 \mathrm{veh} / \mathrm{lane} / \mathrm{km}, a_{m}=$ 1.867 and $v_{\text {free }}=102 \mathrm{~km} / \mathrm{h}$.

Furthermore, we take $\nu_{\text {high }}=65 \mathrm{~km}^{2} / \mathrm{h}, \nu_{\text {low }}=30 \mathrm{~km}^{2} / \mathrm{h}$, and have chosen $a_{\text {speed }}=2$. For the control signals we have assumed that they can change only every minute, and that they cannot be less than $50 \mathrm{~km} / \mathrm{h}$. This is imposed as a hard constraint in the optimization problem. The input of the system is the traffic demand at the upstream end of the link and the

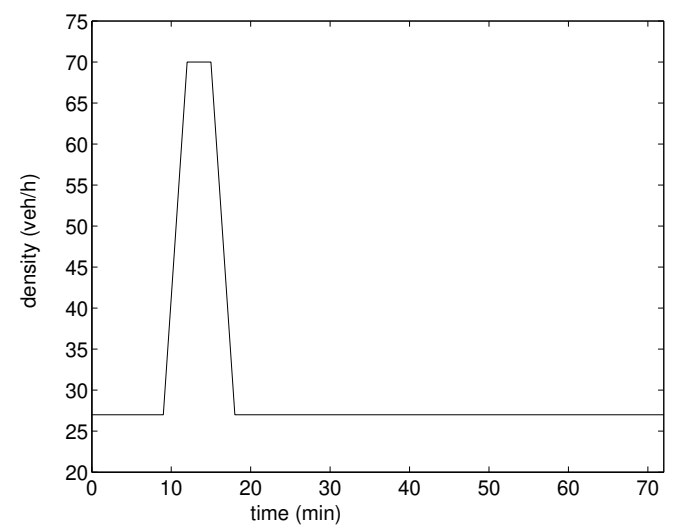

Fioure 4 The downstream densitv scenarin considered in the exneriments

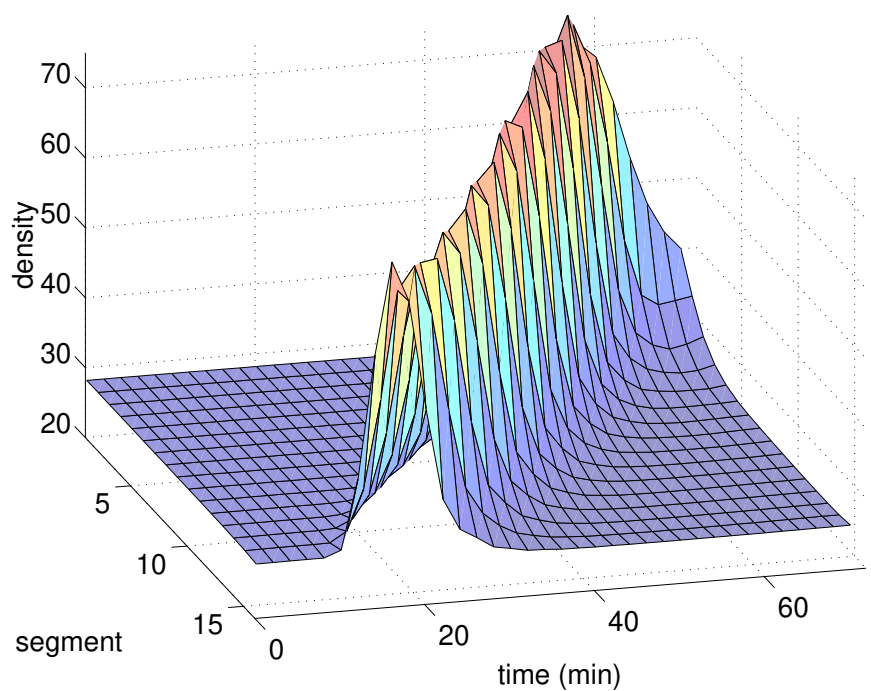

Figure 5. The shock wave propagates through the link in the no control case.

(virtual) downstream density at the downstream end of the link. The traffic demand (inflow) has a constant value of 3900 $\mathrm{veh} / \mathrm{h}$, close to capacity (4000 veh/h). The downstream density equals the steady-state value of $27 \mathrm{veh} / \mathrm{km}$, except for the pulse that represents the shock wave. The pulse was chosen large enough to cause a backpropagating wave in the segments (see Figure 4).

For the above scenario the total time spent (TTS) in the link will be compared for the controlled and uncontrolled cases.

\section{B. Results}

The optimal prediction horizon was found to be approximately $N_{\mathrm{p}}=10 \mathrm{~min}$, which is in the order of the typical travel time through the controlled part of the network (10 $\mathrm{km} / 50 \mathrm{~km} / \mathrm{h}$ ). Shorter predictions horizons did not take the whole response of the system to the speed control into account and resulted in insufficient control actions. Longer prediction horizons tend to take the future demand too much into account, which degrades the performance. A control horizon $N_{\mathrm{c}}=9$ min was necessary. When the difference $N_{\mathrm{p}}-N_{\mathrm{c}}$ was kept constant (1 min), a further increase of $N_{\mathrm{p}}$ caused only a small decrease of the TTS.

The results of the two cases are displayed in Figures 5 and 6 . In the plots we see that in the controlled case the 


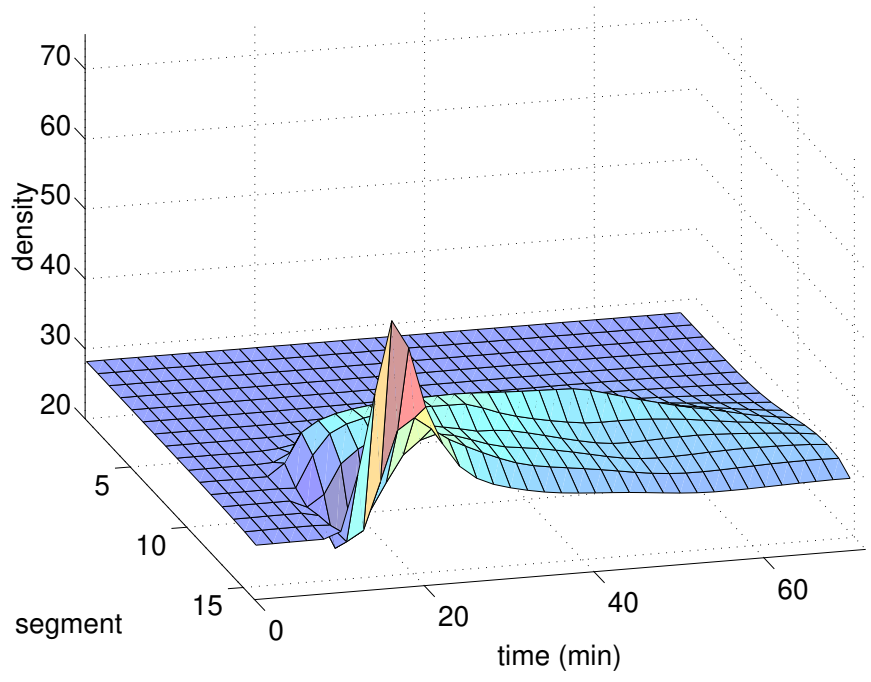

Figure 6. The results for the coordinated control case. The shock wave disappears after 1.5 hour.

shock wave disappears after approximately 1.5 hours, while in the no control case the shock wave travels through the whole link. The active speed limits are in segments 10-14, the others have higher values than the critical speed and are not effective as argued before (see 8). The active speed limits start to limit the flow at $t=10 \mathrm{~min}$ and create a low density wave traveling downstream (the small dip in Figure 6). This low density wave meets the upstream traveling shock wave and reduces its density just enough to stop it. So, the upstream "end" of the shock wave has a fixed location while the downstream "end" dissolves into free flow traffic as in the uncontrolled situation, which means that the shock wave eventually dissolves completely.

The speed limits persist until the shock-wave (to be precise the high density region) is completely dissolved. The speed limits in Figure 8 start to increase after $t=70 \mathrm{~min}$ and (which is not shown here) return to a high value that is not limiting the flow anymore.

The outflow after the shock wave has entered the link is restored earlier to capacity (even temporarily slightly above capacity) in the controlled case (see Figure 7). The TTS was 1321.9 veh.hours in the no control case and 1162.9 veh.hours in the controlled case, which is an improvement of about $12 \%$.

\section{CONCLUSIONS AND FUTURE RESEARCH}

We have applied model predictive control to optimally coordinate variable speed limits. The purpose of the control was to find the control signals that minimize the total time that vehicles spend in the network.

We have applied the developed control framework to a benchmark network consists of a link of $15 \mathrm{~km}$, where the downstream 10 links are controlled by speed limits. The coordinated control case was compared to the no control case. The coordination of speed limits eliminated the shock wave entering from the downstream end of the link. The coordinated case resulted in a network where the outflow was sooner

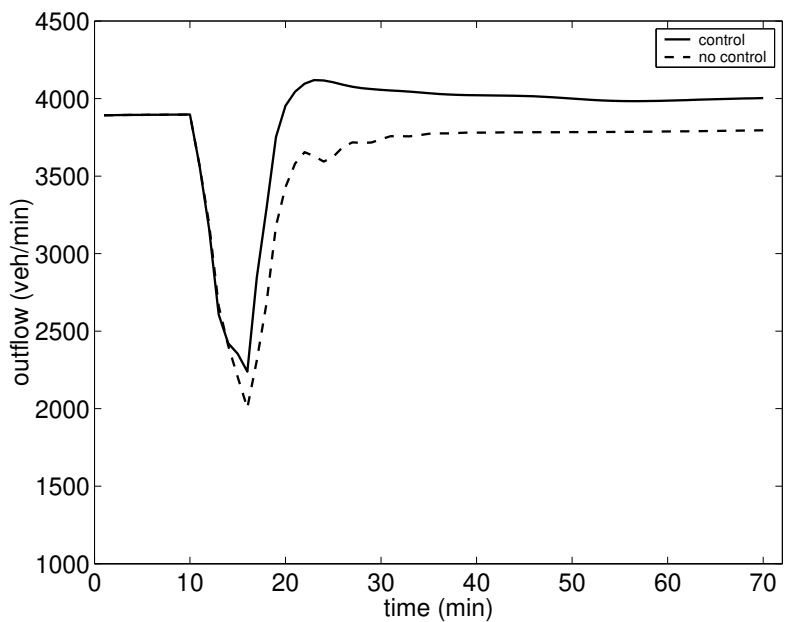

Figure 7. The outflows of the link in the no-control and the coordinated con 110 s. $\mathrm{a} \mathrm{h}$

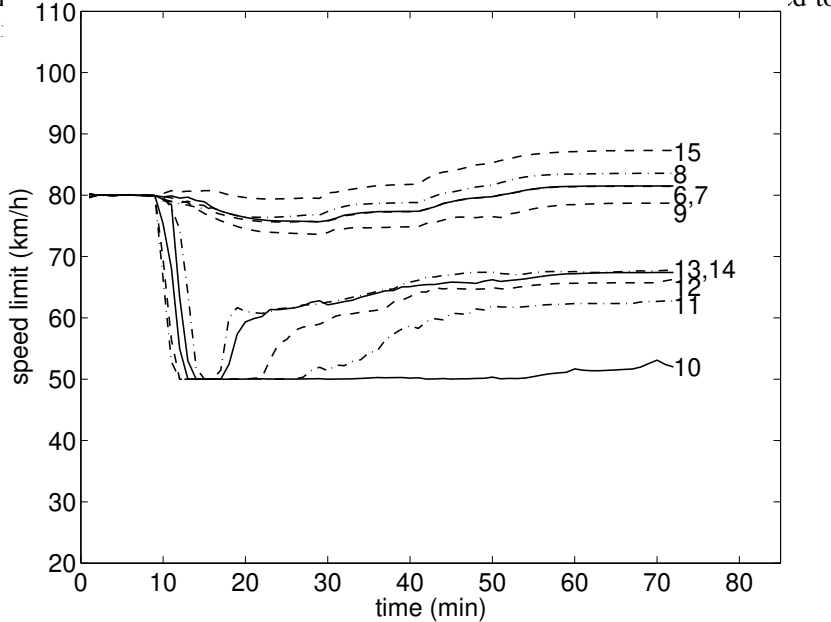

Figure 8. The speed limits during the simulation. The numbers at the lines correspond to the segment numbers. The minimum speed limit is $50 \mathrm{~km} / \mathrm{h}$. restored to capacity (even temporarily above capacity), and in a decrease of the total time spent with $12 \%$.

Topics for further research include: selecting other methods to model the effect of a speed limit; validating the model; further investigation of the effectiveness of MPC for optimal coordination of speed limits for a wider range of scenarios, networks, traffic flow models and/or model parameters; explicit inclusion of modeling errors and disturbances. Furthermore, including extra control measures in addition to speed limits (such as ramp metering, dynamic lane assignment, route information, reversible lanes, etc.) is also a topic for future research.

\section{ACKNOWLEDGMENTS}

This research was supported by the Traffic Research Centre (AVV) of the Dutch Ministry of Transport, Public Works and Water Management.

\section{REFERENCES}

[1] A. Alessandri, A. Di Febbraro, A. Ferrara, and E. Punta. Optimal control of freeways via speed signalling and ramp metering. Control Engineering Practice, 6(6):771-780, June 1998. 
[2] A. Alessandri, A. Di Febbraro, A. Ferrara, and E. Punta. Nonlinear optimization for freeway control using variable-speed signaling. IEEE Transactions on Vehicular Technology, 48(6):2042-2052, November 1999.

[3] E.F. Camacho and C. Bordons. Model Predictive Control in the Process Industry. Springer-Verlag, Berlin, Germany, 1995.

[4] C.C. Chien, Y. Zhang, and P.A. Ioannou. Traffic density control for automated highway systems. Automatica, 33(7):1273-1285, July 1997.

[5] A. Di Febbraro, T. Parisini, S. Sacone, and R. Zoppoli. Neural approximations for feedback optimal control of freeway systems. IEEE Transactions on Vehicular Technology, 50(1):302-312, January 2001.

[6] B.S. Kerner and H. Rehborn. Experimental features and characteristics of traffic jams. Physical Review E, 53(2):R1297-R1300, February 1996.

[7] A. Kotsialos, M. Papageorgiou, and A. Meßmer. Integrated optimal control of motorway traffic networks. In Proceedings of the 1999 American Control Conference (ACC'99), pages 2183-2187, San Diego, California, June 1999.

[8] A. Kotsialos, M. Papageorgiou, and F. Middelham. Optimal coordinated ramp metering with advanced motorway optimal control. In Proceedings of the 80th Annual Meeting of the Transportation Research Board, Washington, DC, 2001. Paper no. 01-3125.

[9] H. Lenz, R. Sollacher, and M. Lang. Nonlinear speed-control for a continuum theory of traffic flow. In Proceedings of the 14th IFAC World Congress (IFAC'99), volume Q, pages 67-72, Beijing, China, January 1999.

[10] H. Lenz, R. Sollacher, and M. Lang. Standing waves and the influence of speed limits. In Proceedings of the European Control Conference 2001 (ECC'01), pages 1228-1232, Porto, Portugal, September 2001.

[11] J.M. Maciejowski. Predictive Control with Constraints. Prentice Hall, Harlow, UK, 2002

[12] M. Papageorgiou. Applications of Automatic Control Concepts to Traffic Flow Modeling and Control. Lecture Notes in Control and Information Sciences. Springer Verlag, Berlin, Germany, 1983.

[13] M. Papageorgiou, J.M. Blosseville, and H. Hadj-Salem. Modelling and real-time control of traffic flow on the southern part of Boulevard Périphérique in Paris: Part I: Modelling. Transportation Research Part A, 24(5):345-359, September 1990.

[14] M. Papageorgiou, J.M. Blosseville, and H. Haj-Salem. Modelling and real-time control of traffic flow on the southern part of Boulevard Périphérique in Paris: Part II: Coordinated on-ramp metering. Transportation Research Part A, 24(5):361-370, September 1990.

[15] S. Smulders. Control of freeway traffic flow by variable speed signs. Transportation Research Part B, 24(2):111-132, April 1990.

[16] E. van den Hoogen and S. Smulders. Control by variable speed signs: Results of the Dutch experiment. In Proceedings of the 7th International Conference on Road Traffic Monitoring and Control, pages 145-149, London, UK, April 1994. 